\title{
ESTRESSES POR SOMBREAMENTO E DESFOLHAMENTO NO RENDIMENTO E SEUS COMPONENTES DA VARIEDADE DE FEIJÃO "CARIOCA"1
}

\section{SHADING AND DEFOLIATION STRESSES ON "CARIOCA" DRY BEANS CULTIVAR YIELD AND COMPONENTS}

\author{
Edelclaiton DAROS ${ }^{2}$ \\ Pedro RONZELLI JÚNIOR ${ }^{3}$ \\ José Antonio COSTA 4 \\ Henrique Soares KOEHLER ${ }^{5}$
}

\begin{abstract}
RESUMO
Foram realizados experimentos, no campo, nos anos agrícolas 1995/96 e 1996/97, na Estação Experimental do Cangüiri, da Universidade Federal do Paraná, Pinhais, PR. O objetivo foi o de entender melhor a interferência de estresses por sombreamento e desfolhamento no mecanismo de compensação da cultura do feijoeiro, variedade 'Carioca', causando redução no rendimento e seus componentes e no índice de colheita aparente. O delineamento experimental utilizado foi o de blocos ao acaso com três repetições, em arranjo fatorial dos tratamentos, $3 \times 7$ [ausência de estresse e estresses por sombreamento e desfolhamento, em sete estádios de desenvolvimento $\left(V_{2}\right.$ a $\left.\left.R_{8}\right)\right]$. O estresse por sombreamento foi simulado com sombrite e o por desfolhamento com o corte das folhas dos feijoeiros. Foram avaliados, nos dois anos, o rendimento e seus componentes (números médios de vagens por planta e de grãos por vagem e massa média de 100 grãos) e o índice de colheita aparente. No ano de 1996/97 os componentes do rendimento foram divididos em procedentes do caule e dos ramos. Os estresses foram responsáveis por alterar a relação fonte-dreno das plantas, refletindo na redução do rendimento, sendo o desfolhamento o que causou maiores perdas. O número médio de vagens por planta foi o componente do rendimento que melhor indicou o efeito dos estresses, sendo o número de vagens do caule mais representativo que o dos ramos, e o índice de colheita aparente foi influenciado pelo desfolhamento em 1995/96 e por desfolhamento e sombreamento em 1996/97. O efeito do estresse por sombreamento foi mais efetivo para interferir no rendimento e seus componentes quando aplicado nos estádios vegetativos e o do desfolhamento nos estádios reprodutivos.
\end{abstract}

Palavras-chave: Phaseolus vulgaris, sombreamento, desfolhamento.

\begin{abstract}
During the growing season of 1995/96 and 1996/97, field experiments were carried out at UFPR Cangüiri Experimental Station, Pinhais, PR. The objective was to better ascertain the influence of shading and defoliation stresses in beans compensation mechanism, cultivar 'Carioca', causing reduction on yield and yield components, and also on the apparent harvest index. The experimental design used was the randomized blocks with three replications, on factorial arrangement of treatments $3 \times 7$ [check and stresses by shading and defoliation on seven development stages $\left(V_{2}\right.$ to $\left.R_{8}\right)$ ]. For the

\footnotetext{
${ }^{1}$ Extraído da tese de Doutorado do primeiro autor.

2 Engenheiro Agrônomo, Dr. Professor Adjunto IV do Curso de Agronomia da UFPR. Rua São Sebastião, 544, CEP 80.540-050, Curitiba, PR. Email: ededaros@agrarias.ufpr.br @ Autor para correspondência.

${ }^{3}$ Engenheiro Agrônomo, CREA 37209/D- 6ª Região, Doutor em Fitotecnia, Universidade Federal do Paraná, Professor Adjunto IV. Caixa Postal 19061, CEP 81.531-990, Curitiba, PR. E-mail: agroprj@agrarias.ufpr.br. Bolsista do CNPq.

${ }^{4}$ Engenheiro Agrônomo, Ph.D., Professor Titular da Faculdade de Agronomia da UFRGS. Bolsista do CNPq.

${ }^{5}$ Engenheiro Florestal, M.Sc., Professor Adjunto IV do Curso de Agronomia da UFPR.
} 
shading stress black shadow screen (sombrite) was used, defoliation was done by cutting the leaves. In both years were evaluated, yield and yield components (averages of pods per plant, seeds per pod, and of 100 seeds weight), and apparent harvest index. Were evaluated in 1996/97 yield components were evaluated separately in stem and branches. The stresses affected the source-sink relationship, resulting in yield reduction, with the highest losses being caused by defoliation. The average pods per plant number was the best indicator of stress effect, being the number of pods on the stem a better indicative than the number of pods on branches. The apparent harvest index was affected by defoliation in 1995/96, and by both defoliation and shading in 1996/97. The stress caused by shading was more effective in causing yield and yield components reduction when applied during the vegetative stages, and the defoliation during the reproductive stages.

Key-words: Phaseolus vulgaris, shading, defoliation.

\section{INTRODUÇÃO}

O feijão, no Brasil, é cultivado em quase todas as áreas, em razão de sua grande adaptação às mais variadas condições climáticas. A cultura do feijoeiro faz parte da maioria dos sistemas produtivos dos pequenos e médios produtores do País, o que faz com que haja baixo rendimento causado pela grande variação de sistemas de produção, tais como consorciado, intercalar e em monocultura. A maior parte do cultivo do feijoeiro é realizada em consórcio com o milho, prática que causa redução da luminosidade para os feijoeiros. Além disso, esse cultivo, muitas vezes, é feito em regiões com baixa quantidade de radiação solar associada com restrições de temperatura e água, fatores que contribuem fortemente para redução da produtividade.

Por outro lado, a perda da área foliar, causada por doenças, insetos, geadas, granizo, vento e déficit hídrico, também interfere diretamente na produtividade do feijoeiro, por alterar suas características fisiológicas. Os efeitos que os estresses provocam nos feijoeiros têm sido estudados com a finalidade de entender as alterações morfológicas que ocorrem nas plantas que, em geral, são responsáveis pela perda de rendimento.

Em relação ao sombreamento artificial em plantas, seu efeito depende, sobretudo, do grau e da duração do sombreamento somados ao estádio de desenvolvimento das plantas (7). Variedades do Tipo II submetidas a $66 \%$ de sombreamento apresentaram redução no rendimento revelada por menor número médio de vagens e grãos por planta, quando esse efeito foi aplicado nos estádios de desenvolvimento $V_{3}, V_{4}$ e $R_{5}(5)$. Também avaliando plantas de variedade do Tipo II, quando o sombreamento foi de 42 e $55 \%$ e aplicado no início da floração $\left(R_{6}\right)$ e estádios subseqüentes, não foram observadas diferenças no rendimento e seus componentes nem no índice de colheita (10), ou quando o sombreamento foi de $0,40,50$ e $76 \%$, durante o florescimento, não foram encontradas diferenças no rendimento (7). Variedades diferentes de feijoeiros dos Tipos I, II, III, em três níveis de luminosidade, 0, 71 e 94\%, tiveram seus rendimentos reduzidos e apresentaram menor número de vagens por planta e de grãos por vagens (16). Outros trabalhos (1, 13, 20 e 22) avaliando diferentes graus de sombreamento em distintos estádios de desenvolvimento de variedades dos tipos II e III também revelaram redução no número de vagens por planta e de grãos por vagem com interferência na redução do rendimento. Da mesma maneira, quando variedades iguais foram semeadas em ambientes de alta e baixa radiação, os maiores rendimentos foram obtidos em região de maior radiação luminosa (14).

Trabalhos de pesquisa simulando diminuição de área foliar por meio de desfolha artificial $(3,6,9$, $11,17,21)$ permitiram verificar que os feijoeiros apresentam diferentes respostas quanto ao hábito de crescimento, ciclo, nível de dano e do estádio em que é aplicado e que, independentemente do hábito de crescimento e do estádio de desenvolvimento, houve influências significativas e negativas sobre o rendimento e seus componentes nos níveis de 66 e 100\% de desfolhamento.

Entendendo que os estresses causados, tanto por sombreamento quanto por desfolhamento, interferem nos componentes do rendimento da cultura do feijoeiro e, conseqüentemente, no seu rendimento, desenvolveu-se o presente trabalho para melhor entender o mecanismo de compensação dos feijoeiros quando esses estresses foram aplicados em diferentes estádios de desenvolvimento.

\section{METODOLOGIA}

O trabalho experimental foi conduzido, no campo, nos anos agrícolas 1995/96 e 1996/97, na Estação Experimental do Cangüiri (EEC), da Universidade Federal do Paraná (UFPR), em Pinhais, PR. O solo da área experimental é um Latossolo Vermelho Amarelo Álico (15), e a região, pela classificação de Köeppen, apresenta clima do tipo Cfb (12). O delineamento experimental empregado nos dois anos foi o de blocos ao acaso 
com três repetições. Os 21 tratamentos testados foram produto do arranjo fatorial de dois tipos de estresses: (a) redução de $50 \%$ de intensidade luminosa em relação à testemunha; e (b) desfolhamento total, mais a testemunha sem estresses, aplicados em sete estádios de desenvolvimento da cultura do feijoeiro: $V_{2}, V_{3}, V_{4}, R_{5}, R_{6}, R_{7}$ e $R_{8}(8)$. Para a redução da intensidade luminosa disponível foram utilizadas telas de plástico preto (sombrite), com vistas a obter retenção de $50 \%$ de luminosidade, fixadas por estacas de madeira a uma altura de 1,00 m do solo, recobrindo toda a parcela. O desfolhamento artificial foi feito com tesouras, cortando-se as folhas dos feijoeiros na base da inserção dos folíolos com o pecíolo, com vistas a obter $100 \%$ de perda de área foliar. Foi utilizada a variedade 'Carioca', do Tipo III, hábito indeterminado prostrado, do grupo diversos, com ciclo médio de 90 dias (2).

O espaçamento utilizado para a semeadura foi o de $0,50 \mathrm{~m}$ entre linhas, tendo as parcelas seis linhas de 6,00 m de comprimento, perfazendo uma área total de $18,00 \mathrm{~m}^{2}$, da qual, consideradas as bordaduras laterais e de cabeceira, foram utilizados $4,00 \mathrm{~m}^{2}$ para avaliação do rendimento e seus componentes e das modificações morfológicas. 0 preparo do solo foi o convencional, com uma aração e duas gradagens. Foi feita adubação com o equivalente a $300 \mathrm{~kg} \cdot \mathrm{ha}^{-1}$ do formulado 4-30-10, colocado no sulco manualmente e incorporado com enxada. A semeadura foi manual e o desbaste realizado no estádio $\mathrm{V}_{2}$. O controle de plantas daninhas foi feito por meio de três capinas manuais. $A$ adubação em cobertura foi feita no estádio $V_{3}$, com o equivalente a $40 \mathrm{~kg} \cdot \mathrm{ha}^{-1}$ de nitrogênio, na forma de uréia. Foram realizadas pulverizações com fungicidas e inseticidas, quando necessário, para o controle de doenças e pragas, que poderiam interferir na redução da área foliar. As principais condições meteorológicas do período dos experimentos podem ser vistas na Tabela 1.

TABELA 1 - Principais condições meteorológicas da região nos períodos de condução dos experimentos. EEC/ UFPR. Pinhais, PR, 1995/96 e 1996/97

\begin{tabular}{|c|c|c|c|c|}
\hline Ano agrícola & $\begin{array}{l}\text { Temperatura média } \\
\text { mínima }\left({ }^{\circ} \mathrm{C}\right)\end{array}$ & $\begin{array}{l}\text { Temperatura média } \\
\text { máxima }\left({ }^{\circ} \mathrm{C}\right)\end{array}$ & $\begin{array}{l}\text { Precipitação total no } \\
\text { ciclo }(\mathrm{mm})\end{array}$ & $\begin{array}{c}\text { Radiação solar média } \\
\text { (cal.cm } \mathrm{cm}^{-2} \text {.D) }\end{array}$ \\
\hline $1995 / 96$ & 13,6 & 26,6 & 599,5 & 398,7 \\
\hline $1996 / 97$ & 12,7 & 23,3 & $1.034,3$ & 329,0 \\
\hline
\end{tabular}

Nos dois anos agrícolas (1995/96 e 1996/97), na maturidade fisiológica dos grãos e considerando a percentagem de umidade em torno de $20 \%$, foram colhidas as plantas das áreas úteis do experimento. Dessas, foram separadas dez plantas para as avaliações dos componentes do rendimento (números médios de vagens por planta e de grãos por vagem e massa média de 100 grãos) e do índice de colheita aparente (ICa). No ano agrícola 1996/ 97, os números médios de vagens e de grãos totais foram divididos, obtendo-se valores desses números para o caule e para os ramos. As demais plantas da área útil da parcela foram trilhadas no campo com trilhadeira estacionária de parcelas e os grãos depois de secos foram avaliados quanto à massa, sendo essa reduzida a $13 \%$ de umidade. As massas dos grãos das dez plantas utilizadas para as avaliações de componentes do rendimento foram somadas às da parcela útil para o cálculo do rendimento final.

Os resultados das avaliações foram submetidos à análise de variância, utilizando-se o programa MSTACT, versão 2.11. As variáveis cujas variâncias mostraram-se homogêneas, pelo teste de Bartlett, tiveram os tratamentos analisados por meio do teste de F. Quando estes tratamentos mostraram-se significativos, tanto a $5 \%$ quanto a $1 \%$ de probabilidade, as médias foram comparadas pelo teste de Tukey, a $5 \%$ de probabilidade $(18,19)$.

\section{RESULTADOS E DISCUSSÃO}

As informações meteorológicas constantes da Tabela 1 permitem verificar que o ano agrícola 1995/96 foi melhor que o de 1996/97 para todas as variáveis avaliadas da cultura do feijoeiro. Destacase a excessiva precipitação pluviométrica ocorrida em ambos os anos, principalmente nesse último período. De qualquer modo, não se pode atribuir, exclusivamente, às condições climáticas quaisquer das diferenças observadas.

Os resultados dos efeitos dos tratamentos sobre o rendimento da cultura do feijoeiro da variedade 'Carioca' são apresentados na Tabela 2. Nota-se, por essa tabela, que o rendimento, nos dois anos agrícolas, foi estatística e significativamente mais prejudicado pelo desfolhamento que pelo sombreamento. No ciclo 1995/96, o estresse por sombreamento causou redução média de 34\%, e de $37 \%$ no de 1996/97. Já o estresse por desfolhamento causou $73 \%$ de redução nos dois ciclos. Alguns pesquisadores $(5,14,16,20)$ também encontraram prejuízos no rendimento como conseqüência do estresse por sombreamento, porém, outros $(7,10)$ discordam desses resultados.

Vê-se, também pela Tabela 2, que para o estresse por sombreamento, nos dois anos agrícolas, houve gradiente de redução do rendimento 
DAROS, E. et al. Estresses por Sombreamento...

TABELA 2 - Rendimento $(R)$ da cultura do feijoeiro, variedade 'Carioca', submetida à ausência de estresse (T), estresse por sombreamento (S) e estresse por desfolhamento (D) associados com sete estádios de desenvolvimento $\left(\mathrm{V}_{2}\right.$ a $\left.\mathrm{R}_{8}\right)$. EEC/UFPR. Pinhais, PR, 1995/96 e 1996/971

\begin{tabular}{|c|c|c|c|c|c|c|c|c|}
\hline \multirow{2}{*}{$\mathrm{R}(\mathrm{kg} / \mathrm{ha})$} & & \multicolumn{7}{|c|}{ Estádios } \\
\hline & & $\mathrm{V}_{2}$ & $V_{3}$ & $V_{4}$ & $\mathrm{R}_{5}$ & $\mathrm{R}_{6}$ & $\mathrm{R}_{7}$ & $\mathrm{R}_{8}$ \\
\hline \multirow{6}{*}{$1995 / 96$} & \multirow[b]{2}{*}{$\mathrm{T}$} & $1.670 \mathrm{a}$ & $1.658 \mathrm{a}$ & $1.692 \mathrm{a}$ & $1.657 \mathrm{a}$ & $1.677 \mathrm{a}$ & $1.700 \mathrm{a}$ & $1.707 \mathrm{a}$ \\
\hline & & A & A & A & A & A & A & A \\
\hline & \multirow[b]{2}{*}{$S$} & $847 b$ & $1.097 \mathrm{~b}$ & $1.053 b$ & $1.180 b$ & $1.003 b$ & $1.203 b$ & $1.383 b$ \\
\hline & & C & BC & BC & $\mathrm{AB}$ & BC & $\mathrm{AB}$ & A \\
\hline & \multirow[b]{2}{*}{$D$} & $608 \mathrm{c}$ & $625 c$ & $517 \mathrm{c}$ & $498 \mathrm{c}$ & $312 c$ & $325 c$ & $263 c$ \\
\hline & & A & A & $A B$ & $A B$ & B & B & B \\
\hline \multirow{6}{*}{$1996 / 97$} & \multirow[b]{2}{*}{$\mathrm{T}$} & $1.630 \mathrm{a}$ & $1.608 \mathrm{a}$ & $1.632 \mathrm{a}$ & $1.670 \mathrm{a}$ & $1.713 \mathrm{a}$ & $1.713 \mathrm{a}$ & $1.700 \mathrm{a}$ \\
\hline & & A & A & A & A & A & A & A \\
\hline & \multirow[b]{2}{*}{$\mathrm{S}$} & $960 \mathrm{~b}$ & $775 b$ & $1.007 b$ & $1.090 \mathrm{~b}$ & $960 \mathrm{~b}$ & $1.167 b$ & $1.383 b$ \\
\hline & & $\mathrm{BC}$ & C & BC & B & $B C$ & $\mathrm{AB}$ & A \\
\hline & \multirow[b]{2}{*}{ D } & $518 \mathrm{c}$ & $477 \mathrm{c}$ & $570 \mathrm{c}$ & $480 \mathrm{c}$ & $380 \mathrm{c}$ & $413 c$ & $347 c$ \\
\hline & & A & A & A & A & A & A & A \\
\hline
\end{tabular}

1 Médias seguidas da mesma letra, minúscula na vertical e maiúscula na horizontal, não diferem significativamente pelo teste de Tukey $(p<0,05)$.

TABELA 3 - Número médio de vagens por planta (NMVP) [1995/96 e 1996/97], número médio de vagens no caule (NMVC) e número médio de vagens nos ramos (NMVR) [1996/97], da cultura do feijoeiro, variedade 'Carioca', submetida à ausência de estresse (T), estresse por sombreamento (S) e estresse por desfolhamento (D) associados com sete estádios de desenvolvimento $\left(\mathrm{V}_{2}\right.$ a $\left.\mathrm{R}_{8}\right)$. EEC/UFPR. Pinhais, $\mathrm{PR}{ }^{1}$

\begin{tabular}{|c|c|c|c|c|c|c|c|c|}
\hline \multirow[b]{2}{*}{ Variáveis } & & \multicolumn{7}{|c|}{ Estádios } \\
\hline & & $\mathrm{V}_{2}$ & $V_{3}$ & $\mathrm{~V}_{4}$ & $\mathrm{R}_{5}$ & $\mathrm{R}_{6}$ & $\mathrm{R}_{7}$ & $\mathrm{R}_{8}$ \\
\hline \multirow{6}{*}{$\begin{array}{c}\text { NMVP } \\
1995 / 96\end{array}$} & \multirow[b]{2}{*}{$\mathrm{T}$} & $17,1 \mathrm{a}$ & $17,7 \mathrm{a}$ & $17,1 \mathrm{a}$ & $17,4 \mathrm{a}$ & $17,3 a$ & $17,5 a$ & $17,7 \mathrm{a}$ \\
\hline & & A & A & A & A & A & A & A \\
\hline & \multirow[b]{2}{*}{ S } & $7,1 \mathrm{~b}$ & $9,6 \mathrm{~b}$ & $8,7 \mathrm{~b}$ & $10,1 b$ & $8,3 b$ & $9,2 \mathrm{~b}$ & $12,5 b$ \\
\hline & & C & $\mathrm{BC}$ & $\mathrm{BC}$ & $\mathrm{AB}$ & $\mathrm{BC}$ & $\mathrm{BC}$ & A \\
\hline & \multirow[b]{2}{*}{ D } & $5,1 \mathrm{c}$ & $6,5 \mathrm{c}$ & $4,8 \mathrm{C}$ & $5,7 \mathrm{c}$ & $3,4 \mathrm{c}$ & $3,8 \mathrm{c}$ & $3,6 \mathrm{c}$ \\
\hline & & $\mathrm{AB}$ & A & $A B$ & $\mathrm{AB}$ & B & $\mathrm{AB}$ & B \\
\hline \multirow{6}{*}{$\begin{array}{c}\text { NMVP } \\
1996 / 97\end{array}$} & \multirow[b]{2}{*}{$\mathrm{T}$} & $16,23 \mathrm{a}$ & $16,43 \mathrm{a}$ & $14,93 \mathrm{a}$ & $15,03 \mathrm{a}$ & $15,46 \mathrm{a}$ & $14,70 \mathrm{a}$ & $15,33 \mathrm{a}$ \\
\hline & & A & A & A & A & A & A & A \\
\hline & \multirow[b]{2}{*}{ S } & $6,80 \mathrm{~b}$ & $7,93 \mathrm{~b}$ & $8,23 b$ & $8,56 \mathrm{~b}$ & $8,26 \mathrm{~b}$ & $8,76 \mathrm{~b}$ & $13,23 b$ \\
\hline & & B & B & B & B & B & B & A \\
\hline & \multirow[b]{2}{*}{ D } & $5,76 b$ & $5,76 \mathrm{c}$ & $5,83 \mathrm{c}$ & $3,30 \mathrm{c}$ & $3,20 \mathrm{c}$ & $4,10 \mathrm{c}$ & $5,16 \mathrm{c}$ \\
\hline & & A & A & A & A & A & A & A \\
\hline \multirow{6}{*}{$\begin{array}{l}\text { NMVC } \\
1996 / 97\end{array}$} & \multirow[b]{2}{*}{$\mathrm{T}$} & 6,66 a & $6,56 \mathrm{a}$ & $6,36 a$ & $6,20 a$ & 5,96 a & $5,80 a$ & $6,60 \mathrm{a}$ \\
\hline & & A & A & A & A & A & A & A \\
\hline & \multirow{3}{*}{ S } & $4,76 \mathrm{~b}$ & $5,13 \mathrm{~b}$ & $4,26 b$ & $4,36 \mathrm{~b}$ & $4,60 \mathrm{~b}$ & $4,13 \mathrm{~b}$ & $4,76 b$ \\
\hline & & A & A & A & A & A & A & A \\
\hline & & $3,96 \mathrm{~b}$ & $4,10 \mathrm{~b}$ & $3,66 b$ & $2,13 \mathrm{c}$ & $1,60 \mathrm{c}$ & $1,80 \mathrm{c}$ & $2,70 \mathrm{c}$ \\
\hline & D & A & A & $\mathrm{A}$ & B & B & B & $\mathrm{AB}$ \\
\hline \multirow{6}{*}{$\begin{array}{c}\text { NMVR } \\
1996 / 97\end{array}$} & \multirow{2}{*}{$\mathrm{T}$} & $9,56 a$ & $9,55 a$ & $8,56 a$ & $8,83 a$ & $9,50 \mathrm{a}$ & $9,03 a$ & $9,26 a$ \\
\hline & & A & A & A & A & A & A & A \\
\hline & \multirow{2}{*}{$S$} & $2,03 \mathrm{~b}$ & $2,80 \mathrm{~b}$ & $3,93 \mathrm{~b}$ & $4,20 \mathrm{~b}$ & $3,80 \mathrm{~b}$ & $4,33 \mathrm{~b}$ & $8,43 b$ \\
\hline & & C & BC & BC & BC & BC & B & C \\
\hline & D & $1,80 \mathrm{~b}$ & $1,66 \mathrm{~b}$ & $2,23 b$ & $1,16 \mathrm{c}$ & $1,60 \mathrm{c}$ & $2,30 \mathrm{c}$ & $2,50 \mathrm{c}$ \\
\hline & D & A & A & A & A & A & A & A \\
\hline
\end{tabular}

Médias seguidas da mesma letra, minúscula na vertical e maiúscula na horizontal, não diferem significativamente pelo teste de Tukey $(p<0,05)$. 
estatisticamente significativo, da ordem de $39 \%$, em 1995/96, e de 44\%, em 1996/97, mostrando que esse estresse é mais efetivo para causar perdas quando ocorre nos estádios iniciais ou vegetativos. Quanto ao estresse por desfolhamento, observou-se ação inversa, ou seja, é mais prejudicial quando ocorre nos estádios reprodutivos. Neste caso, foram encontradas diferenças estatísticas significativas no ciclo de 1995/ 96 , quando a redução no rendimento foi da ordem de 58\%. Em 1996/97, mesmo sem diferenças estatísticas significativas, a redução no rendimento foi da ordem de $40 \%$. Resultados da avaliação do estresse pelo desfolhamento obtidos por outros autores $(3,6,17$, 21) confirmam que, independentemente dos estádios estudados, níveis de 66 a 100\% de desfolhamento são responsáveis por redução no rendimento.

$\mathrm{Na}$ Tabela 3 são apresentados os resultados referentes aos números médios de vagens por planta [1995/96 e 1996/97] e das divisões por caule e ramos
[1996/97]. Observa-se, por essa tabela, que o número médio de vagens na planta, nos dois anos agrícolas, foi estatística e significativamente mais prejudicado pelo desfolhamento que pelo sombreamento, repetindo o observado para o rendimento e confirmando a importância desse componente para o rendimento (4). A variação percentual da redução causada pelos estresses foi também e neces-sariamente semelhante à observada para o rendimento. Da mesma forma, a semelhança pode ser verificada quanto aos estresses por sombreamento e desfolhamento ao longo dos estádios de desenvolvimento. O gradiente de redução repete-se como mais efetivo nos estádios vegetativos, para o sombreamento, e nos reprodutivos, para o desfolhamento. Resultados da diminuição do número médio de vagens por planta, em função tanto do efeito do sombreamento $(5,13,16,22)$ quanto do desfolhamento $(6,9,11,17,21)$, foram observados e confirmam os encontrados.

TABELA 4 - Número médio de grãos por vagem (NMSV [1995/96 e 1996/97], número médio de grãos por vagem no caule (NMSVC) e número médio de grãos por vagem nos ramos (NMSVR) [1996/97], da cultura do feijoeiro, variedade 'Carioca', submetida à ausência de estresse $(T)$, estresse por sombreamento (S) e estresse por desfolhamento (D) associados com sete estádios de desenvolvimento $\left(V_{2}\right.$ a $\left.R_{8}\right)$. EEC/UFPR. Pinhais, $P R^{1}$

\begin{tabular}{|c|c|c|c|c|c|c|c|c|}
\hline \multirow[b]{2}{*}{ Variáveis } & & \multicolumn{7}{|c|}{ Estádios } \\
\hline & & $V_{2}$ & $V_{3}$ & $V_{4}$ & $\mathrm{R}_{5}$ & $R_{6}$ & $R_{7}$ & $\mathrm{R}_{8}$ \\
\hline \multirow{6}{*}{$\begin{array}{c}\text { NMSV } \\
1995 / 96\end{array}$} & \multirow{3}{*}{$\mathrm{T}$} & $4,9 a$ & $5,1 \mathrm{a}$ & $5,2 \mathrm{a}$ & $5,0 \mathrm{a}$ & $4,9 a$ & $5,1 a$ & $5,1 \mathrm{a}$ \\
\hline & & $A$ & $\mathrm{~A}$ & $\mathrm{~A}$ & $A$ & $A$ & $A$ & $A$ \\
\hline & & $4,7 \mathrm{a}$ & $4,1 \mathrm{~b}$ & $4,3 \mathrm{~b}$ & $4,2 \mathrm{~b}$ & $4,2 \mathrm{~b}$ & $4,8 \mathrm{a}$ & $4,8 a$ \\
\hline & S & $A B C$ & C & $A B C$ & $\mathrm{BC}$ & $A B C$ & A & A \\
\hline & \multirow[b]{2}{*}{ D } & $2,8 \mathrm{~b}$ & $2,2 \mathrm{c}$ & $2,6 \mathrm{c}$ & $3,1 \mathrm{c}$ & $3,1 \mathrm{c}$ & $2,5 \mathrm{~b}$ & $3,4 \mathrm{~b}$ \\
\hline & & $A B C$ & $D$ & $\mathrm{BCD}$ & $A B C$ & $A B C$ & $C D$ & A \\
\hline \multirow{6}{*}{$\begin{array}{l}\text { NMSV } \\
1996 / 97\end{array}$} & \multirow{2}{*}{$\mathrm{T}$} & $4,56 \mathrm{a}$ & $4,59 \mathrm{a}$ & $4,69 \mathrm{a}$ & $4,64 a$ & $4,49 a$ & $4,57 \mathrm{a}$ & $4,69 a$ \\
\hline & & A & A & A & A & A & A & A \\
\hline & \multirow[b]{2}{*}{$S$} & $4,26 a$ & $3,95 a$ & $3,98 \mathrm{a}$ & $3,71 b$ & $3,76 a b$ & $3,99 \mathrm{a}$ & $3,87 \mathrm{a}$ \\
\hline & & A & A & A & A & A & A & A \\
\hline & \multirow[b]{2}{*}{ D } & $2,84 \mathrm{~b}$ & $2,74 b$ & $2,68 \mathrm{~b}$ & $2,79 \mathrm{c}$ & $3,12 b$ & $2,86 b$ & $3,23 \mathrm{~b}$ \\
\hline & & A & A & A & A & A & A & A \\
\hline \multirow{6}{*}{$\begin{array}{l}\text { NMSVC } \\
1996 / 97\end{array}$} & \multirow[b]{2}{*}{$\mathrm{T}$} & $4,73 a$ & $4,68 \mathrm{a}$ & $4,91 \mathrm{a}$ & $4,77 \mathrm{a}$ & $4,85 \mathrm{a}$ & $4,67 \mathrm{a}$ & $4,88 \mathrm{a}$ \\
\hline & & A & A & A & A & A & A & A \\
\hline & \multirow{3}{*}{ S } & $4,31 \mathrm{a}$ & $3,88 \mathrm{a}$ & $4,20 \mathrm{a}$ & $3,71 \mathrm{~b}$ & $4,22 \mathrm{a}$ & $4,45 \mathrm{a}$ & $4,19 \mathrm{a}$ \\
\hline & & A & A & A & A & A & A & A \\
\hline & & $2,84 b$ & $2,50 \mathrm{~b}$ & $2,71 \mathrm{~b}$ & $2,92 b$ & $3,05 b$ & $2,96 \mathrm{~b}$ & $3,37 \mathrm{~b}$ \\
\hline & D & A & A & A & A & A & A & A \\
\hline \multirow{6}{*}{$\begin{array}{l}\text { NMSVR } \\
1996 / 97\end{array}$} & \multirow[b]{2}{*}{$\mathrm{T}$} & $4,40 a$ & $4,52 \mathrm{a}$ & $4,47 \mathrm{a}$ & $4,51 \mathrm{a}$ & $4,14 a$ & $4,46 \mathrm{a}$ & $4,50 \mathrm{a}$ \\
\hline & & A & A & A & A & A & A & A \\
\hline & \multirow[b]{2}{*}{$S$} & $4,20 \mathrm{a}$ & $4,02 \mathrm{a}$ & $3,76 \mathrm{a}$ & $3,72 \mathrm{a}$ & $3,30 a b$ & $3,53 \mathrm{a}$ & $3,56 a b$ \\
\hline & & A & A & A & A & A & A & A \\
\hline & \multirow[b]{2}{*}{ D } & $2,88 b$ & $3,00 \mathrm{a}$ & $2,64 \mathrm{~b}$ & $2,66 \mathrm{~b}$ & $3,20 \mathrm{~b}$ & $2,76 \mathrm{~b}$ & $3,10 \mathrm{~b}$ \\
\hline & & A & A & A & A & A & A & A \\
\hline
\end{tabular}

1 Médias seguidas da mesma letra, minúscula na vertical e maiúscula na horizontal, não diferem significativamente pelo teste de Tukey $(p<0,05)$. 
DAROS, E. et al. Estresses por Sombreamento...

TABELA 5 - Índice de colheita aparente (ICa) da cultura do feijoeiro, variedade 'Carioca', submetida a três tratamentos: ausência de estresse (T), estresse por sombreamento (S) e estresse por desfolhamento (D), em sete estádios de desenvolvimento. EEC/UFPR. Pinhais, PR, 1995/96 e 1996/97

\begin{tabular}{|c|c|c|c|c|c|c|c|c|}
\hline \multirow[b]{2}{*}{$\mathrm{ICa}$} & & \multicolumn{7}{|c|}{ Estádios } \\
\hline & & $V_{2}$ & $V_{3}$ & $V_{4}$ & $\mathrm{R}_{5}$ & $\mathrm{R}_{6}$ & $\mathrm{R}_{7}$ & $\mathrm{R}_{8}$ \\
\hline \multirow{6}{*}{ 1995/96 } & & $0,58 a$ & $0,56 a$ & $0,59 a$ & $0,56 a$ & $0,58 a$ & $0,57 \mathrm{a}$ & $0,59 a$ \\
\hline & T & A & A & A & A & A & A & A \\
\hline & & $0,51 \mathrm{a}$ & $0,48 \mathrm{a}$ & $0,49 a$ & $0,49 a$ & $0,50 \mathrm{a}$ & $0,50 \mathrm{a}$ & $0,51 \mathrm{a}$ \\
\hline & s & A & A & A & A & A & A & A \\
\hline & & $0,40 \mathrm{~b}$ & $0,38 b$ & $0,36 \mathrm{~b}$ & $0,35 b$ & $0,40 \mathrm{~b}$ & $0,34 b$ & $0,38 \mathrm{~b}$ \\
\hline & D & $\mathrm{A}$ & $\mathrm{A}$ & A & $\mathrm{A}$ & $\mathrm{A}$ & $\mathrm{A}$ & A \\
\hline \multirow{6}{*}{$1996 / 97$} & & $0,55 \mathrm{a}$ & $0,54 \mathrm{a}$ & $0,54 a$ & $0,56 \mathrm{a}$ & $0,57 \mathrm{a}$ & $0,58 \mathrm{a}$ & $0,58 \mathrm{a}$ \\
\hline & $\mathrm{T}$ & A & A & A & A & A & A & A \\
\hline & & $0,46 b$ & $0,46 b$ & $0,48 \mathrm{~b}$ & $0,46 \mathrm{~b}$ & $0,50 \mathrm{~b}$ & $0,51 b$ & $0,49 b$ \\
\hline & S & A & A & A & A & A & A & A \\
\hline & & $0,38 \mathrm{c}$ & $0,29 c$ & $0,34 \mathrm{c}$ & $0,21 \mathrm{c}$ & $0,33 \mathrm{c}$ & $0,27 c$ & $0,34 \mathrm{c}$ \\
\hline & D & A & $\mathrm{BC}$ & $\mathrm{AB}$ & C & $\mathrm{AB}$ & $\mathrm{BC}$ & $A B$ \\
\hline
\end{tabular}

1 Médias seguidas da mesma letra, minúscula na vertical e maiúscula na horizontal, não diferem significativamente pelo teste de Tukey $(p<0,05)$.

Na mesma Tabela 3, observa-se que a divisão do número médio de vagens totais da planta em vagens oriundas do caule e dos ramos mantém o comportamento em relação aos estresses relatado para o rendimento e permite verificar que as reduções são mais drásticas quando se analisam os números referentes às vagens dos ramos em que o sombreamento representou $54 \%$ de redução e o desfolhamento $79 \%$, em comparação com os do caule, que foram, respectivamente, 28 e $55 \%$.

$\mathrm{Na}$ Tabela 4 são apresentados os resultados referentes aos números médios de grãos por vagem [1995/96 e 1996/97] e às divisões por caule e ramos [1996/97]. Observa-se, por essa tabela, que esse componente também mantém o comportamento em relação aos estresses relatado para o rendimento, ou seja, o desfolhamento foi mais prejudicial que o sombreamento. No entanto, vê-se que a redução foi percentualmente menor nos dois anos agrícolas, da ordem de 12 e 15\%, respectivamente, para 1995/96 e 1996/97, para o sombreamento, e de 44 e $37 \%$, para o desfolhamento. Nota-se também que os efeitos diluíram-se quase proporcionalmente na divisão, indicando que esse componente do rendimento é de menor importância quando comparado com o número médio de vagens por planta. Estes resultados encontrados confirmam os encontrados por outros autores para sombreamento $(1,5,20)$ e desfolhamento (6).

Quanto à massa de 100 grãos, foi observado, nos dois anos agrícolas, que os resultados não mostraram diferenças estatísticas significativas. Isso vem confirmar que, dos componentes do rendimento, esse é o que menor influência tem sobre o rendimento, uma vez que a característica é de alta herdabilidade (21).
Na Tabela 5 são apresentados os resultados referentes ao índice de colheita aparente (ICa) nos dois anos agrícolas. Observa-se, por essa tabela, que no ano 1995/96 houve efeito estatisticamente significativo do desfolhamento com redução média no valor do índice da ordem $36 \%$. No ano 1996/97 foram observados efeitos dos dois estresses, sendo o desfolhamento mais danoso que o sombreamento. A redução média no valor do índice foi, respectivamente, de 45 e $14 \%$.

\section{CONCLUSÕES}

1) Os estresses por sombreamento e desfolhamento alteram a relação fonte-dreno das plantas, com reflexos na redução do rendimento, em ambos os anos, sendo o desfolhamento o que causou maiores perdas.

2) Os números médios de vagens por planta e de grãos por vagem foram os componentes do rendimento efetivamente influenciados pelos estresses, destacando-se o número médio de vagens por planta.

3) O número médio de vagens no caule é melhor indicador do rendimento que o número médio de vagens nos ramos.

4) O índice de colheita aparente foi influenciado pelo desfolhamento em 1995/96 e por desfolhamento e sombreamento em 1996/97.

5) O efeito do estresse por sombreamento foi mais efetivo para interferir no rendimento e seus componentes quando aplicado nos estádios vegetativos e o do desfolhamento nos estádios reprodutivos. 


\section{REFERÊNCIAS}

01 AGUIAR NETO, A.O.; MOURA, E.G.; SOUZA, V.F.; BARBOSA, E.M.; BARBOSA, M.D.; CAIRES, E.F.; RODRIGUES, J.D.; PEDRAS, J.F.; ONO, E.O. Efeito do sombreamento e adubação nitrogenada no crescimento e na produção da cultura do feijoeiro (Phaseolus vulgaris L.). Científica, São Paulo, v.23, n.2, p.209-221, 1995.

02 ALMEIDA, L.D.A.; LEITÃO FILHO, H.; MIYASAKA, S. Características do feijão Carioca, um novo cultivar. Bragantia, Campinas, v.30, p.33-38, 1977.

03 APPADURAI, R.R.; RAJAKARUNA, S.B. Leaf contribution to pod yields in kidney bean (Phaseolus vulgaris L.). Indian Journal Agricultural Science, New Delhi, v.37, p.524-525, 1967.

04 BENNET, J.P.; ADAMS, M.W.; BURGA, C. Pod yield component variation and intercorrelation in Phaseolus vulgaris L. as affected by planting density. Crop Science, Madison, v.17, p.73-75, 1977.

05 CHAGAS, J.M. Efeitos do desfolhamento artificial sobre três cultivares de feijão (Phaseolus vulgaris L). Viçosa, 1977. 44 f. Tese (Doutorado em Agronomia) - Curso de Pós-Graduação em Fitotecnia, Universidade Federal de Viçosa.

06 DAROS, E.; RONZELLI JÚNIOR, P.; PISSAIA, A.; ZAMBON, J.L.C.; KOEHLER, H.S. Efeitos do desfolhamento sobre os componentes do rendimento e características morfológicas do feijoeiro. II. Cultivar 'IAPAR 8 - RIO NEGRO'. Revista do Setor de Ciências Agrárias, Curitiba, v.10, n.1/2, p.119-123, 1988.

07 ESCALANTE-ESTRADA, J.A.S; KOHASHI-SHIBATA, J. Efecto del sombreamento artificial sobre algunos parametros del crescimento en frijól (Phaseolus vulgaris L.). Agrociencia, Chapingo, v.48, p.29-38, 1982.

08 FERNÁNDES, F.; GEPTS, P.; LOPES, M. Etapas de desarollo en la planta de frijol. In: LÓPEZ, M.; FERNÁNDEZ, F.; SCHOONHOVEN, A. (Eds.) FRIJOL: Investigación e produción. Cali: CIAT, 1985. p.61-78.

09 GALVEZ, G.E.; GALINDO, J.J.; ALVAREZ, G. Defolación artificial para estimar pérdidas por daños foliares em fríjol (Phaseolus vulgaris L.). Turrialba, San Jose, v.27, n.2, p.143-146, 1977.

10 GÓMEZ-RAMIREZ, B. Ensayo preliminar del efecto de sombreado en postfloración sobre los componentes del rendimento en frijol (Phaseolus vulgaris L.). Morelos: 1976. Tesis (Licenciatura en Agronomia) - Escuela de Ciências Biológicas, Universidad Autónoma del Estado de Morelos.

11 HOHMANN; C.L.; CARVALHO, S.M. Efeito de redução foliar sobre o rendimento do feijoeiro (Phaseolus vugaris L. 1753). American Society of Entomology, Brasil, v.12, n.1, p.3-9, 1983.

12 INSTITUTO AGRONÔMICO DO PARANÁ. Cartas climáticas do Estado do Paraná. Londrina: IAPAR, 1994. 49p.

13 LOPES, N.F.; OLIVA, M.A.; CARDOSO, M.J.; GOMES, M.M.S.; SOUZA, V.F. Crescimento e conversão da energia solar em feijão (Phaseolus vulgaris L.) submetido a três densidades de fluxo radiante e dois regimes hídricos. Revista Ceres, Viçosa, v.33, n.186, p.142-144, 1986.

14 MAGALHÃES A.C.; MONTOJOS, J.C. Effect of solar radiation on the growth parameters and yield of two varieties of common beans (Phaseolus vulgaris L.). Turrialba, San Jose, v.21, n.2, p.165-168, 1971.

15 OLMOS-ITURRI, L.J.; CARDOSO, A.; CARVALHO, A.P.; HOCHMÜLER, D.P.; FASOLO, P.J.; RAÜEN, M.J. Levantamento de reconhecimento dos solos do Estado do Paraná. Curitiba: EMBRAPA/SNLCS/SUDESUL/IAPAR, 1984. 412 p. (Boletim Técnico, 57)

16 PORTES, T.A.; CARVALHO, J.R.P. Área foliar, radiação solar, temperatura do ar e rendimento em consorciação e em monocultivo de diferentes cultivares de milho e feijão. Pesquisa Agropecuária Brasileira, Brasília, v.18, n.7, p.755-762, 1983.

17 RONZELLI JÚNIOR, P., DAROS, E.; PISSAIA, A.; ZAMBON, J.L.C.; KOEHLER, H.S. Efeitos do desfolhamento sobre os componentes do rendimento e características morfológicas do feijoeiro. I. Cultivar 'Carioca'. Revista do Setor de Ciências Agrárias, Curitiba, v.10, n.1/2, p.113-117, 1988.

18 SNEDECOR, G.W.; COCHRAN, W.G. Statistical methods. 7th ed. Ames: The lowa State University Press, 1980. 507 $\mathrm{p}$.

19 STEEL, R. G. D.; TORRIE, J. H. Principles and procedures of statistics with special reference to the biolocical sciences. New York: McGrow-Hill Co., 1960. 481p.

20 TORQUEBIAU, E.; AKYEAMPONG, E. Shedding some ligth on shade: its effects on beans, maize and bananas. Agroforestry Today, v.6, n.4, p.14-15, 1994.

21 VIEIRA, C. Effect of artificial defolation on the yield of two indeterminate bean (Phaseolus vulgaris L.) cultivars. Turrialba, San Jose, v.31, n.4, p.383-385, 1981.

22 XIA, M.Z. Effect of shading on nodule growth and seed yield in faba bean (Vicia faba L.). Tropical Agriculture, Guildford, v.72, n.4, p.290-296, 1995. 